\title{
Revisão Integrativa dos Diagnósticos de Enfermagem de Pacientes em Período Pós Operatório
}

\section{Integrative Review of Nursing Diagnoses of Patients in Postoperative Period}

\author{
Kátia Cilene Godinho Bertoncello*; ${ }^{a *}$ Bruna Sávio ${ }^{a}$ Juliana Martins Ferreira ${ }^{\text {b }}$ Eliane Regina Pereira do Nascimento ${ }^{\mathrm{c}}$ \\ a'Universidade Federal de Santa Catarina, Departamento de Enfermagem, Programa de Mestrado Profissional Multidisciplinar em Saúde, SC, Brasil \\ ${ }^{\mathrm{b}}$ Clinica Dircksen, SC, Brasil \\ 'Universidade Federal de Santa Catarina. Departamento de Enfermagem, SC, Brasil \\ *E-mail: kbertoncello@yahoo.com.br \\ Recebido: 10 de junho de 2014; Aceito: 24 de novembro de 2014
}

\begin{abstract}
Resumo
A prática baseada em evidências é uma abordagem que preconiza a utilização de resultados de pesquisas na prática clínica, sendo a revisão integrativa um de seus recursos. Dessa forma, tal processo foi empregado neste estudo com o objetivo de descrever a produção científica dos Diagnósticos de Enfermagem atribuídos aos pacientes em período pós-operatório nos últimos seis anos. Para a seleção dos artigos, utilizou-se a base de dados de Literatura Latino-Americana em Ciências de Saúde (LILACS), no período de janeiro de 2006 até março de 2012 . A amostra desta revisão se constituiu de cinco artigos, selecionados em três etapas: leitura dos títulos, leitura dos resumos e dos que se encontravam disponíveis na íntegra em meio eletrônico. Dentro os artigos encontrados, tangentes aos principais diagnósticos dos pacientes em pósoperatório, constituindo o tema foco, foram encontrados os seguintes diagnósticos de enfermagem: Risco para infecção, Déficit de autocuidado para banho e Dor. Conclui-se com essa investigação, que a produção de artigos que tratam da temática Diagnósticos de Enfermagem para os pacientes em pós-operatório é pequena no Brasil, e ausente com ênfase nos Diagnósticos de Enfermagem para os pacientes em pós-operatório, não apenas para atender a nova legislação em vigor a respeito da Sistematização da Assistência de Enfermagem, ,mas porque o profissional Enfermeiro é um dos principais responsáveis no atendimento inicial desses pacientes e necessita de ferramentas que potencializem este cuidado individualizado.
\end{abstract}

Palavras-chave: Diagnóstico de Enfermagem. Enfermagem em Pós-Anestésico. Enfermagem Perioperatória.

\begin{abstract}
The evidence-based practice is an approach focused on research findings in clinical practice, with an integrative review. Thus, this study aimed at describing the scientific production of nursing diagnoses assigned to patients in the postoperative period in the last six years. For the selection of articles, the database of Latin American Literature in Health Sciences (LILACS), from January 2006 until March 2012 was used. Five articles were selected in three stages: reading the titles, the abstracts, and those available in their entirety in the electronic media. The following nursing diagnoses were found: Risk for infection, self-care deficit bathing and pain. It is concluded that the production of articles that deal with the theme Nursing Diagnoses for patients in the postoperative period is small, in Brazil and absent in Latin America and the Caribbean during the period investigated. There is need for further research, with emphasis on nursing diagnoses for patients in the postoperative period, not only to meet the new legislation regarding the Systematization of Nursing Care, but also because the professional nurse is a major contributor in the initial care of these patients and requires tools that enhance this individualized care.
\end{abstract}

Keyword: Nursing Diagnosis. Postanesthesia Nursing. Perioperative Nursing.

\section{Introdução}

A cirurgia, para qualquer pessoa, é uma situação estressante e complexa e a sua história vem de muito tempo. Desde a préhistória, já eram realizados procedimentos cirúrgicos, porém acredita-se que a maioria deles não tinha objetivo terapêutico e sim de expulsão de espíritos malignos, visto que essa era a crença naquela época ${ }^{1}$.

Com o passar do tempo, surgiram as escolas médicas e, posteriormente, os cirurgiões-barbeiros, que realizavam procedimentos, como sangrias, drenagem de abscessos entre outros, sem nenhum cuidado com higiene ou processos anestésicos, o que levava a grande número de infecções e morte das pessoas ${ }^{2}$.

A taxa de mortalidade começou a diminuir no século XIX, com a melhora da higiene, sendo que uma das principais responsáveis foi à enfermeira Florence Nightingale que, durante o atendimento aos feridos na Guerra da Criméia, conseguiu diminuir as infecções, devido a suas medidas de limpeza, alimentação e vigilância ${ }^{2}$.

Os procedimentos cirúrgicos vêm evoluindo a cada dia, se tornando um procedimento mais seguro, com maior exatidão dos diagnósticos pré-operatórios e segurança na indicação cirúrgica, com terapia e planejamento da técnica cirúrgica, o que contribuiu para um pós-operatório mais rápido e melhor para os pacientes ${ }^{2}$.

A enfermagem perioperatória é uma especialidade que abrange vários aspectos do cuidado em enfermagem. A atuação do enfermeiro nesse período é efetuar uma avaliação 
em cada uma das fases, que compreende o pré-operatório, o intra-operatório e o pós operatório ${ }^{3}$.

A Sistematização da Assistência de Enfermagem SAE eleva a qualidade da assistência de enfermagem, o que beneficia o paciente, visto que o atendimento é individualizado ${ }^{4}$. Porém, na prática assistencial, o Processo de Enfermagem - $\mathrm{PE}^{4}$ não vem sendo usado como deveria, pelas enfermeiras, devido a diversas justificativas, como a dificuldade de decisão com base no raciocínio clínico diagnóstico, falta de tempo, conhecimento e até mesmo por falta de preocupação com a quantidade dos registros e planejamento do cuidado de enfermagem ${ }^{5}$.

A viabilização do cuidado de enfermagem se faz por meio do PE, que é a dinâmica das ações sistematizadas e inter/relacionadas, visando à assistência ao ser humano. Caracteriza-se pelo inter/relacionamento e dinamismo de suas fases ou passos. O PE tem sido considerado uma possibilidade de resposta a sérios questionamentos referentes à qualidade do cuidado e desenvolvimento científico da enfermagem ${ }^{6}$.

Entre as cinco etapas do Processo de Enfermagem, destacam-se a segunda, o chamado Diagnóstico de Enfermagem - DE, tema desta investigação. Essa expressão passou a ser utilizada formalmente na década de 50, no contexto de diagnósticos clínicos. Em 1973, realizou-se uma conferência internacional para identificar as necessidades dos pacientes que estivessem no âmbito da enfermagem, estabelecendo termos descritivos que pudessem ser utilizados no mundo todo, os chamados diagnósticos de enfermagem ${ }^{5}$.

Desde então, os DE, representam a apreciação clínica das respostas do indivíduo, da família ou da comunidade, aos problemas de saúde/processos existenciais reais ou potenciais, portanto é um julgamento quanto à necessidade ou um problema que requer intervenções de enfermagem e tratamento ${ }^{7}$.

Diante da necessidade de atender a Resolução do COFEN $n^{\circ} 358 / 2009$, que dispõe sobre a Sistematização da Assistência de Enfermagem e implementação do Processo de Enfermagem em todos os ambientes, públicos ou privados, em que ocorre o cuidado do profissional de Enfermagem, o PE deve ser realizado de modo deliberado e sistemático, sendo obrigatório o seu registro formal de todas as suas fases no prontuário do paciente ${ }^{8}$.

Dessa forma, considerando a relevância do assunto e, principalmente, a necessidade constante de atualização na área, justifica-se o desenvolvimento deste estudo que teve a seguinte questão norteadora: quais os Diagnósticos de Enfermagem dos pacientes em período pós-operatório, publicados na literatura nos últimos seis anos?

Frente a esta nova problemática e esperando contribuir para melhora da prática de enfermagem perioperatória através do uso de diagnósticos de enfermagem, a presente investigação teve como objetivo descrever a produção cientifica dos Diagnósticos de Enfermagem atribuídos aos pacientes em período pós-operatório nos últimos seis anos.

\section{Desenvolvimento}

\subsection{Metodologia}

Este método constitui um instrumento da Prática Baseada em Evidências, o que mantém, pois, os profissionais mais informados para a tomada de decisões, tornando as práticas de saúde mais eficazes ${ }^{9}$. Além disso, possibilita uma compreensão abrangente dos problemas pertinentes aos cuidados de saúde, sejam os relacionados à enfermagem, impacto da doença ou tratamento do paciente ${ }^{10}$.

A Prática Baseada em Evidências é uma abordagem que preconiza a utilização de resultados de pesquisas na atividade clínica. A revisão integrativa, um de seus recursos, é empregada neste estudo com o objetivo de descrever as evidências disponíveis sobre os fatores presentes no processo de enfermagem no pós-operatório, com foco principal nos diagnósticos.

Desse modo, a revisão integrativa aprofunda a busca por trabalhos que ofereçam subsídios mais concretos para comparação das características e processos que envolvam os cuidados prestados ao paciente em pós-operatório. Esse método mundialmente reconhecido auxilia a sintetizar os resultados de pesquisas relevantes, o que proporciona troca e ampliação do conhecimento, formando ideias mais consolidadas e fundamentadas para o exercício profissional ${ }^{6}$.

Para construção da revisão integrativa, foram percorridas seis etapas: seleção das palavras-chave; definição da base de dados para busca; estabelecimento dos critérios para seleção da amostra; análise geral dos resultados da busca; construção e preenchimento de um formulário para registro dos dados coletados; análise dos dados e interpretação dos resultados?

Procurou-se identificar a procedência, o tipo de estudo, os descritores, o objetivo da pesquisa, a metodologia, os resultados e a sua relação com a temática deste trabalho, sintetizando, assim, a amostra para análise. Os resultados foram codificados com números e identificados com o título do trabalho.

Optou-se, como base de dados para realização da pesquisa, a Literatura Latino- Americano em Ciências de Saúde LILACS, sendo selecionados apenas os trabalhos publicados dentro do período compreendido entre janeiro de 2006 até março de 2012. A busca foi realizada nos meses de abril e maio de 2012. Através dos Descritores em Ciências da Saúde - DeCS, foram selecionados seis descritores para pesquisa: Diagnóstico de Enfermagem; Registros de Enfermagem; Planejamento de Assistência ao Paciente; Processos de Enfermagem; Enfermagem Perioperatória e Enfermagem em Pós-Anestésico.

A partir destes descritores, realizaram-se 10 cruzamentos, associando-os entre si, sendo excluídos os cruzamentos repetidos ou com resultado nulo. A partir desses cruzamentos, seis se mostraram produtivos para a constituição da amostra, de acordo com os seguintes critérios: textos na forma de artigos, teses e dissertações disponíveis na íntegra e que abordaram de alguma forma o tema "diagnóstico de enfermagem no 
paciente em período pós-operatório", nos idiomas português, espanhol ou inglês.

A seleção da amostra foi realizada em três etapas. A primeira deu-se pela leitura dos títulos dos trabalhos, sendo excluídos os que não tivessem relação com o tema. Desses, posteriormente, foram lidos os resumos, com o intuito de uma maior aproximação e conhecimento do trabalho. Após essa seleção, buscaram-se os textos que se encontravam disponíveis na íntegra, que foram analisados por meio dos dados registrados nos formulários (Figura 1).

Figura 1: Fluxograma relativo às etapas de seleção dos artigos

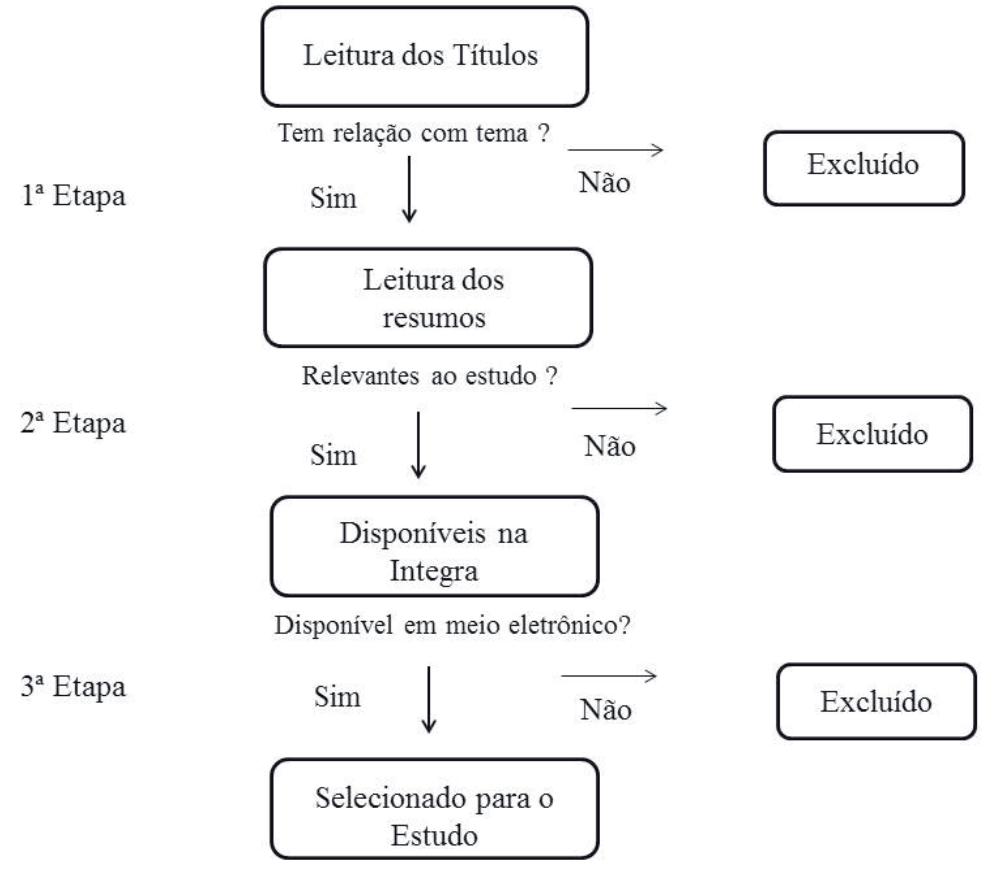

\subsection{Discussão}

Dentro dos seis cruzamentos que constituíram a amostra, foram encontradas 609 publicações (Tabela 1). $\mathrm{Na}$ primeira análise, considerando o título do trabalho, foram selecionados 17 estudos, todos artigos científicos.
Posteriormente, foram selecionados sete artigos após a leitura dos resumos. Na busca pelos trabalhos completos, dois não estavam disponíveis no meio eletrônico. Desta forma, a amostra final foi constituída de cinco artigos científicos.

Tabela 1: Resultados obtidos na base de dados LILACS. Florianópolis, 2012

\begin{tabular}{|c|c|c|c|c|c|c|c|c|c|c|}
\hline \multirow{3}{*}{ Cruzamento de descritores } & \multicolumn{2}{|c|}{$\begin{array}{c}\text { Trabalhos } \\
\text { Encontrados }\end{array}$} & \multicolumn{6}{|c|}{ Excluídos/Critérios de exclusão } & \multicolumn{2}{|c|}{ Selecionados } \\
\hline & \multirow{2}{*}{$\mathbf{N}^{\circ}$} & \multirow{2}{*}{$\%$} & \multicolumn{2}{|c|}{ Fora do período } & \multicolumn{2}{|c|}{ Repetidos } & \multicolumn{2}{|c|}{ Fora do tema } & \multirow{2}{*}{$\mathbf{N}^{\mathbf{0}}$} & \multirow{2}{*}{$\%$} \\
\hline & & & $\mathbf{N}^{\mathbf{0}}$ & $\%$ & $\mathbf{N}^{0}$ & $\%$ & $\mathbf{N}^{\mathbf{0}}$ & $\%$ & & \\
\hline 1. Diagnóstico de Enfermagem & 547 & 89,8 & 354 & 64,7 & 4 & 0,7 & 180 & 32,9 & 9 & 1,6 \\
\hline 2. Enfermagem em Pós-Anestésico & 31 & 5 & 20 & 64,5 & $=$ & - & 7 & 22,5 & 4 & 12,9 \\
\hline $\begin{array}{l}\text { 3. Enfermagem Perioperatória e } \\
\text { Diagnóstico de Enfermagem }\end{array}$ & 19 & 3,1 & 13 & 68,4 & $=$ & - & 2 & 10,5 & 4 & 21 \\
\hline $\begin{array}{l}\text { 4. Enfermagem Perioperatória e } \\
\text { Registros de Enfermagem }\end{array}$ & 6 & 0,9 & 3 & 50 & 2 & 33,3 & 1 & 16,6 & $=$ & - \\
\hline $\begin{array}{l}\text { 5. Enfermagem Perioperatória e } \\
\text { Planejamento de Assistência ao Paciente }\end{array}$ & 4 & 0,6 & 3 & 75 & 1 & 25 & $=$ & - & $=$ & - \\
\hline $\begin{array}{l}\text { 6. Enfermagem Perioperatória e } \\
\text { Processos de Enfermagem }\end{array}$ & 2 & 0,3 & 2 & 100 & $=$ & - & $=$ & - & $=$ & - \\
\hline Total & 609 & 100 & 395 & 64 & 7 & 1,1 & 190 & 31,1 & 17 & 2,8 \\
\hline
\end{tabular}

Fonte: Dados da pesquisa

Diante dessa primeira análise, com seis cruzamentos, o descritor que mais apresentou publicações foi Diagnóstico de Enfermagem ${ }^{11}$, com 89,8\% dos trabalhos encontrados, sendo que o descritor que teve o segundo maior índice foi 
Enfermagem em Pós-Anestésico, com 5\%.

Os outros cruzamentos juntos tiveram um pouco mais de $5 \%$ dos trabalhos encontrados. Com os critérios de exclusão (fora do período, repetidos e fora do tema), foram excluídos cerca de $97 \%$ dos trabalhos encontrados, sendo que o critério de exclusão mais significativo (64\% dos casos) foi estar fora do período pré determinado (ter mais de 5 anos de publicação), seguido de fora do tema (31\%) e repetidos (1\%).

Em uma segunda análise, através dos resumos dos trabalhos, foram selecionados sete artigos, que mostraram maior relevância para o tema "diagnóstico de enfermagem no pós-operatório", através de três cruzamentos de descritores, Diagnóstico de Enfermagem (três artigos), descritor mais significativo na primeira análise, Enfermagem perioperatória e Diagnóstico de Enfermagem (três artigos) e Enfermagem em Pós-Anestésico (um artigo).

Por fim, a amostra final foi composta por cinco artigos (Quadro 1), visto que dois dos artigos selecionados anteriormente não estavam disponíveis na íntegra em meio eletrônico.

Quadro 1: Artigos selecionados de acordo com periódico, local e ano de publicação (LILACS)

\begin{tabular}{|l|c|c|c|}
\hline \multicolumn{1}{|c|}{ Título do artigo } & Periódico & Ano & Instituição do autor principal \\
\hline $\begin{array}{l}\text { 1. Diagnósticos de enfermagem em pacientes submetidos à } \\
\text { cirurgia cardíaca }^{12}\end{array}$ & $\begin{array}{c}\text { Revista Brasileira de } \\
\text { Enfermagem }\end{array}$ & 2006 & Hospital Messejana - Ceará \\
\hline $\begin{array}{l}\text { 2. Transplante renal: diagnósticos e intervenções de } \\
\text { enfermagem em pacientes no pós-operatório imediato }\end{array}$ & Einstein & 2007 & $\begin{array}{c}\text { Hospital Israelita Albert Einstein São } \\
\text { Paulo }\end{array}$ \\
\hline $\begin{array}{l}\text { 3. Diagnósticos De Enfermagem De Pacientes Em Período } \\
\text { Pós-Opperatório Imediato de Colecistectomia Laparoscópica }\end{array}$ & $\begin{array}{c}\text { Revista Latino-americana } \\
\text { de Enfermagem }\end{array}$ & 2006 & $\begin{array}{c}\text { Escola de Enfermagem de Ribeirão } \\
\text { Preto }\end{array}$ \\
\hline $\begin{array}{l}\text { 4. Pacientes Transplantados Renais: análise de associação } \\
\text { dos diagnósticos de enfermagem }\end{array}$ & $\begin{array}{c}\text { Revista Gaúcha de } \\
\text { Enfermagem }\end{array}$ & 2010 & Universidade Federal do Ceará \\
\hline $\begin{array}{l}\text { 5. Diagnóstico de Enfermagem em pacientes pós-cateterismo } \\
\text { cardíaco - contribuição de Orem }\end{array}$ & $\begin{array}{c}\text { Revista Brasileira de } \\
\text { Enfermagem }\end{array}$ & 2006 & $\begin{array}{c}\text { Escola de Enfermagem da } \\
\text { Universidade Federal de Minas Gerais }\end{array}$ \\
\hline
\end{tabular}

Fonte: Dados da pesquisa

Os cinco artigos selecionados são oriundos das Regiões Sudeste e Nordeste do Brasil, tendo uma pequena predominância $(60 \%)$ de publicações da Região Sudeste, quadro que corresponde com a distribuição das Universidades no país, uma vez que a Região Sudeste conta com $48 \%$ das Universidades do Brasil, seguida pela Região Sul e Nordeste (17\%), Centro-Oeste $(10 \%)$ e Norte $(5 \%)$, o que, de certa forma, justificaria a distribuição das publicações ${ }^{13}$. Porém, se forem considerados os estados e não a região, esse panorama muda completamente, onde o Estado do Ceará tem $60 \%$ das publicações.

Cabe ressaltar que foi surpreendente não encontrar artigos de outros países latinos. Entende-se que o motivo possa ter sido o período investigado, ou seja, os últimos seis anos, pois a base de dados (LILACS) utilizada para busca contém artigos científicos de 27 países Latino Americanos e do Caribe.

No que diz respeito ao tipo de estudo, quatro artigos foram pesquisas descritivas exploratórias, do tipo transversal e um estudo de caso fundamentado na Teoria do Déficit do Autocuidado. Houve uma predominância (80\%) de estudos com delineamento das pesquisas quantitativas, sendo que apenas o estudo de caso apresentou abordagem qualitativa. A maioria dos estudos mostrou uma abordagem quantitativa.

Em relação à metodologia, as técnicas de coletas de dados foram diversificadas, incluindo entrevista clínica e exame físico e consulta de prontuários, instrumento com base no modelo conceitual de Horta. Porém, de certa forma, houve uma uniformidade nos procedimentos de coleta de dados, já que em todos os trabalhos, independente do instrumento utilizado para a investigação, a entrevista clínica era realizada junto com o exame físico, para obter os diagnósticos de enfermagem e desenvolver uma das etapas do PE.

Analisando os sujeitos da pesquisa, os cinco trabalhos utilizaram pacientes submetidos a algum tipo de cirurgia. Em quatro dos trabalhos, os pacientes se encontravam em pós-operatório imediato e apenas um trabalho apresentou pacientes em atendimento cirúrgico ambulatorial.

Essas pesquisas foram realizadas em hospitais, visto que se tratava de pacientes em pós-operatório que, em geral, são tratados em unidades hospitalares. Houve predominância (80\%) de hospitais públicos, sendo que dois eram hospitais universitários e um era hospital privado.

Quanto aos objetivos dos artigos, todos traziam claramente a objetividade relacionada aos diagnósticos de enfermagem. Em dois deles, o objetivo estava em usar os diagnósticos de enfermagem para planejar a assistência de enfermagem, diante das intervenções de enfermagem, segundo o Nursing Intervensions $^{17}$ (NIC). Já, em outros dois, os objetivos se restringiam na identificação dos diagnósticos encontrados com maior frequência para aqueles pacientes, e um único trabalho buscou analisar os diagnósticos de enfermagem em sua integridade, incluindo os fatores relacionados e as características definidoras.

Enfim, no que diz respeito aos resultados encontrados, houve uma diversidade muito grande, o que era esperado, já que todos os estudos tratavam de tipos específicos de cirurgias. Além disso, o tipo de análise foi diferente. Em todos os trabalhos, foi encontrada a lista geral dos diagnósticos de 
enfermagem, porém em dois deles foram analisados apenas os diagnósticos com mais de $50 \%$ de frequência, outro apenas os com mais de $75 \%$ de frequência e os demais apenas analisaram os diagnósticos com $100 \%$ de presença.

Em um dos artigos foram encontrados 19 DE, sendo que apenas 15 foram analisados por terem mais de 50\% de frequência. No outro artigo, que utilizava o mesmo tipo de análise, foram encontrados $18 \mathrm{DE}$ e analisados 8 . O artigo que se deteve aos $75 \%$ de frequência, foram analisados 10 de 38 diagnósticos. Nos demais, foram identificados 25 e 9 diagnósticos presentes em 100\% dos pacientes. Com base em todos os diagnósticos analisados nos artigos, portanto que estavam presentes em mais de $50 \%$ dos pacientes, pode-se observá-los na lista dos 21 diagnósticos, porém nenhum deles estava presente nos cinco artigos e alguns em apenas um dos casos (Quadro 2).

Quadro 2: Diagnósticos de Enfermagem analisados por número de trabalhos que o citaram

\begin{tabular}{|l|c|}
\hline \multicolumn{1}{|c|}{ Diagnóstico de Enfermagem } & $\begin{array}{c}\text { Presença em } \\
\text { trabalhos }\end{array}$ \\
\hline Risco para infecção & 4 \\
\hline Déficit de autocuidado - banho & 3 \\
\hline Dor & 3 \\
\hline Risco para Integridade da pele prejudicada & 2 \\
\hline Mobilidade prejudicada & 2 \\
\hline Risco para desequilíbrio de temperatura & 2 \\
\hline Integridade tissular prejudicada & 2 \\
\hline Percepção sensorial prejudicada - visão e audição & 2 \\
\hline Risco de aspiração & 2 \\
\hline Nutrição desequilibrada & 2 \\
\hline Risco para função respiratória alterada & 1 \\
\hline Hipotermia & 1 \\
\hline Risco para Nutrição desequilibrada & 1 \\
\hline Padrão de sono prejudicado & 1 \\
\hline Fadiga & 1 \\
\hline Disfunção sexual & 1 \\
\hline Risco para lesão orgânica renal & 1 \\
\hline Risco para constipação & 1 \\
\hline Integridade da pele prejudicada & 1 \\
\hline Risco para volume de líquido desequilibrado & 1 \\
\hline Risco de queda & \\
\hline
\end{tabular}

Fonte: Dados da pesquisa

Diante desse quadro, podemos considerar como Diagnósticos de Enfermagem predominantes nos pacientes em pós-operatórios: Risco de infecção; e Déficit de autocuidado para banho e Dor, já que esses estão presentes em mais de $50 \%$ dos trabalhos.

O Diagnóstico de Risco de infecção, que foi encontrado em quatro artigos, é definido como risco aumentado de ser invadido por organismos patógenos ${ }^{11}$. As defesas primárias, destruição de tecidos, trauma, procedimentos invasivos, doenças crônicas e exposição ambiental aumentada a patógenos foram os fatores de risco encontrados.

Nos pacientes em pós-operatório, é comum o rompimento do tecido epitelial, devido à incisão cirúrgica, que dificulta a chegada de nutrientes e oxigênio aos tecidos, facilitando a ocorrência de um processo infeccioso, além dos procedimentos invasivos realizados na sala de cirurgia, como colocação de drenos, punção venosa para terapia de fluidos entre outros, juntamente com as defesas primárias reduzidas devido aos anestésicos ${ }^{18}$.

A identificação dos principais fatores de risco envolvidos nesse diagnóstico de Risco de infecção instrumentaliza o enfermeiro a tomar medidas preventivas, visando a interromper esse ciclo recorrente de possível infecção hospitalar, pois a intervenção direta e completa no paciente em pós-operatório pode influenciar o seu bom prognóstico ou não ${ }^{19}$.

O DE, Déficit no autocuidado para banho, teve relação com Dor em $100 \%$ dos pacientes em pós-operatório, manifestado por incapacidade de acessar o banheiro, lavar o corpo, obter a fonte da água, pegar artigos para o banho, regular a água do banho ou secar o corpo ${ }^{11}$.

A dor é uma experiência sensorial e emocional desagradável, com caráter subjetivo, sendo que seu significado, para o indivíduo, está relacionado com as experiências vividas durante o procedimento cirúrgico. A dor é uma das principais queixas encontradas nos pacientes em pós-operatório, tanto em pacientes de origem clínica, quanto nos pacientes cirúrgicos de urgência, apresentando manifestações com intensidade moderada e forte, sendo esta a mais comum ${ }^{20}$.

O Diagnóstico de Enfermagem de Dor tem como definição a experiência sensorial e emocional desagradável que surge de lesão tissular real ou potencial ou descrita em termos de tal lesão; tem início súbito ou lento e intensidade leve a intensa, com término antecipado ou previsível e duração menor que seis meses ${ }^{11}$.

Nos artigos, o Diagnóstico de Dor foi relacionado a agentes lesivos e descrito por característica definidora de expressão facial de dor, alteração na pressão sanguínea, mudança na frequência cardíaca e dilatação pupilar ${ }^{11}$.

$\mathrm{O}$ não cuidado da dor aguda leva à ativação de vias neuronais por um período, prolongando as disfunções orgânicas e os efeitos prejudiciais, como aumento da frequência cardíaca e respiratória, levando a uma diminuição da distribuição do oxigênio nos tecidos, diminuição da perfusão sanguínea periférica e contração muscular reflexa, consequentemente interferindo na recuperação pós-operatória dos pacientes ${ }^{20}$.

\section{Conclusão}

A revisão integrativa é um método de pesquisa que ainda precisa ser mais utilizado na enfermagem, uma vez que sua contribuição é muito valiosa ao fornecer subsídios científicos 
para melhoria do cuidado prestado aos pacientes.

Pode-se concluir com essa investigação que a produção de artigos que tratam da temática Diagnósticos de Enfermagem para os pacientes em pós-operatório é pequena no Brasil e ausente na América Latina e Caribe no período investigado. Entretanto, pode-se identificar que os Diagnósticos de Enfermagem mais encontrados foram: Risco para infecção e Déficit de autocuidado para banho e Dor.

Há necessidade de novas pesquisas, com ênfase nos Diagnósticos de Enfermagem para os pacientes em pósoperatório, não apenas para atender a nova legislação em vigor a respeito da Sistematização da Assistência de Enfermagem, mas porque o profissional Enfermeiro é um dos principais responsáveis no atendimento inicial desses pacientes e necessita de ferramentas que potencializem um cuidado individualizado. Dessa forma, ao incorporar evidências clínicas na prática da Enfermagem, benefícios são oferecidos à população, ao profissional e ao sistema.

\section{Referências}

1. Smeltzer SC, Bare BG. Hinkle JL, Cheever KH. Bruner. Tratado de enfermagem médico cirúrgica. Rio de Janeiro: Guanabara Koogan; 2011.

2. Ferreira E, Borges HV, Figueiredo MSD. Visita pré-operatória: um instrumento para o cuidar do enfermeiro de centro cirúrgico. 2007. 71f. Monografia [Trabalho de Conclusão de Curso] - Universidade Federal de Santa Catarina; 2007.

3. Calicchio LG. Práticas recomendadas - SOBECC: Centro Cirúrgico, Recuperação Pós Anestésica, Centro de Materiais e Esterilização. Paulo: SOBECC; 2009.

4. Cunha SMB, Barros ALBL. Análise da implementação da Sistematização da Assistência de Enfermagem, segundo o Modelo Conceitual de Horta. Rev Bras Enferm 2005;58(5):568-72.

5. Andrade JS, Vieira, MJ. Prática assistencial de enfermagem: problemas, perspectivas e necessidade de sistematização. Rev Bras Enferm 2005;58(3):261-5.

6. Galdeano LE, Rossi LA. Construção e validação de instrumentos de coleta de dados para o período perioperatório de cirurgia cardíaca. Rev Latinoam Enferm 2002;10(6):800-4.

7. Doenges ME, Moorhouse MF, Murr AC. Diagnósticos de enfermagem. Rio de Janeiro: Guanabara Koogan; 2009.
8. Conselho Federal de Enfermagem. Dispõe sobre a Sistematização da Assistência de Enfermagem e a implementação do Processo de Enfermagem em ambientes, públicos ou privados, em que ocorre o cuidado profissional de Enfermagem, e dá outras providências. Resolução nº358, de 15 de outubro de 2009.

9. Webster P. Evidence based practice - what is it and how can it be encouraged in orthopaedic nursing? J Orthop Nurs 2002;6(3):140-3.

10. Souza MT, Silva MD, Carvalho R. Revisão integrativa: o que é e como fazer. Einstein 2010;8(1):102-6.

11. North American Nursing Diagnosis Association. Diagnóstico de enfermagem da Nanda: definições e classificação 20122014. Porto Alegre: Artmed; 2013.

12. Rocha LA, Maia TF, Silva LF. Diagnósticos de enfermagem em pacientes submetidos à cirurgia cardíaca. Rev Bras Enferm 2006;59(3):321-6.

13. Luvisotto MM, Carvalho R, Galdeano LE. Transplante renal: diagnósticos e intervenções de enfermagem em pacientes no pós-operatório imediato. Einstein 2007;5(2):117-22.

14. Dalri CC, Rossi LA, Dalri MCB. Diagnósticos de enfermagem de pacientes em período pós-operatório imediato de colecistectomia laparoscópica. Rev Latinoam Enferm 2006;14(3):389-96.

15. Lira ALBC, Lopes MVO. Pacientes transplantados renais: análise de associação dos diagnósticos de enfermagem. Rev Gaúcha Enferm 2010;31(1):108-14.

16. Lima LR, Pereira SVM, Chianca TCM. Diagnósticos de enfermagem em pacientes pós-cateterismo cardíacocontribuição de Orem. Rev Bras Enferm 2006;59(3):285-90.

17. Bulechek GM, Butcher HK, Dochterman JM. Classificação das intervenções de enfermagem - NIC. Porto Alegre: Artmed; 2010.

18. Bertoncello KCG, Cavalcanti CD’AK, Ilha P. Diagnósticos reais e proposta de intervenções de enfermagem para os pacientes vítimas de múltiplos traumas. Rev Eletr Enferm 2013;15(4):905-14. doi: 10.5216/ree.v15i4.19497.

19. Bertoncello KCG, Cavalcanti CD'AK, Ilha P, Nascimento ERP do. Diagnósticos de risco e propostas de intervenções de Enfermagem aos pacientes vítimas de múltiplos traumas. Rev Bras Pesq Saúde 2013;15(2):23-31.

20. Silva FS, Fernandes MV, Volpato MP. Diagnósticos de enfermagem em pacientes internados pela clínica ortopédica em unidade médico-cirúrgica. Rev Gaucha Enferm 2008;29(4):565-72. 